\title{
Ion channel distributions in cortical neurons are optimized for energy-efficient active dendritic computations
}

\author{
${ }^{1}$ Max Planck Group: In Silico Brain Sciences, Center of Advanced European Studies and Research, \\ Bonn, Germany. \\ ${ }^{2}$ International Max Planck Research School for Brain and Behavior, Bonn, Germany.
}

\begin{abstract}
${ }^{*}$ Corresponding author. Email: marcel.oberlaender@caesar.de arco.bast@caesar.de
\end{abstract}

The mammalian brain uses more than $20 \%$ of the energy consumed by the entire body. This enormous demand for energy is thought to impose strong selective pressure by which neurons evolve in ways that ensure robust function at minimal energy cost. Here we demonstrate that the ion channel expression patterns by which pyramidal tract neurons - the major output cell type of the cerebral cortex - could implement their complex intrinsic physiology is extremely widespread. Surprisingly, this wide spectrum does not reflect morphological variability, but the energy costs for generating dendritic calcium action potentials. We found that energy-efficient calcium action potentials require a low expression of slow inactivating potassium channels in the distal dendrites, an empirical observation whose significance remained unclear for more than a decade. Thus, cortical neurons do not utilize all theoretically possible ways to implement their functions, but instead appear to select those optimized for energy-efficient active dendritic computations.

\section{Introduction}

It is thought that the neurons in the cerebral cortex evolved to be highly energy efficient in neural computation $^{1,2}$. Most of the cortical energy costs result directly from the generation and transmission of electrical and chemical signals within and between neurons ${ }^{3}$. For example, the initiation of action potentials (APs) in the axon is metabolically expensive, consuming more than $20 \%$ of the energy in the gray matter of the rodent brain ${ }^{4,5}$. Experimental studies ${ }^{6,7}$ and simulations of single compartment models $^{8}$ found that the energy cost for AP generation in mammalian neurons are remarkably close to the theoretically predicted minimum ${ }^{9}$, whereas the energy consumption of APs in invertebrates is far from being optimal ${ }^{8,10}$. These observations indicate that biophysical parameters in the axon of cortical neurons - e.g. the kinetics of ion channels ${ }^{11}$ or the expression patterns of sodium $\left(\mathrm{Na}^{+}\right)$and potassium

$40\left(\mathrm{~K}^{+}\right)$channels ${ }^{12}$ - are optimized for generating APs with minimal energy cost. However, whether optimization of biophysical parameters could represent a general principle by which cortical neurons implement energy-efficient signal processing in the dendrites is unknown.

One major challenge for answering this question is that cortical neurons, and particularly the pyramidal

tract neurons in layer 5 (L5PTs), express a variety of voltage-gated ion channels in specific dendritic domains ${ }^{13,14}$. As a result, L5PTs perform active dendritic computations, for example by generating calcium $\left(\mathrm{Ca}^{2+}\right)$ APs around the main branch point of the apical dendrite ${ }^{15-18}$. Distal dendritic inputs, which normally appear greatly attenuated at the cell body (i.e., soma), must cross a high threshold to evoke calcium APs ${ }^{17}$, but can then generate bursts of axonal APs ${ }^{19}$. Hence, L5PTs can act as cellular 'coincidence detectors' by switching their axonal output from single AP to burst firing when proximal 
and distal inputs are provided synchronously ${ }^{19}$. Thus, while single compartment models are sufficient to assess whether biophysical mechanisms are optimized in the axon for energy-efficient generation of single $\mathrm{APs}^{8}$, deriving such information for signal processing in entire cortical neurons requires multicompartmental models that can account for both the particular morphology of the dendrites and the respective expression patterns of the various ion channels along them.

Previous modelling attempts were able to capture certain aspects of the L5PTs' physiology separately ${ }^{20,21}$. However, generating models of L5PTs that capture their dendritic, perisomatic, and axonal physiology has been a long-standing challenge. To the best of our knowledge, a model accomplishing this task is currently only available for one dendritic morphology reconstructed from an in vitro labeled L5PT ${ }^{13}$. The ion channel distributions derived for this morphology failed, however, to reproduce the physiology observed for other morphologies ${ }^{22}$. Therefore, it is assumed that L5PTs and neurons in general - establish proper function by compensating morphological variability with variability in the expression of ion channels ${ }^{23}$. However, conclusive answers to the questions - Which principles govern the relationships between dendrite morphology, ion channel expression and neuronal function, and could energy efficiency be one of these principles? - remain unknown.

Here we quantitatively address these questions by generating millions of biophysically detailed multicompartmental models for in vivo labeled L5PTs that we obtained from the vibrissa-related part of rat primary somatosensory cortex ${ }^{24-26}$ - the barrel cortex. We demonstrate that these models represent the spectrum of theoretically possible combinations of morphology and ion channel expression that can give rise to the characteristic functions of L5PTs. By analyzing this comprehensive dataset of biophysically, morphologically and physiologically realistic neuron models, we show that morphology only weakly constrains ion channel distributions and densities. Instead, energy efficient generation of the dendritic calcium AP imposes strong constraints for the expression of ion channels.

\section{Results}

We generated multi-compartmental models for 12 in vivo labeled neurons (Fig. S1a) that represent the morphological variability across L5PTs in the rat barrel cortex (Fig. S1b). For this purpose, we used the biophysical parameters reported by Hay et al., ${ }^{13}$, representing those that yielded so far the only acceptable L5PT model. Moreover, we modified the genetic algorithm that Hay et al., ${ }^{13}$ developed for parameter fitting to be suitable for parameter space exploration (Methods). This modification allowed us to investigate which expression patterns of a diverse set of ion channels (Table S1) - i.e., their respective distributions and densities in the soma, axon, basal and apical dendrites - could implement the intrinsic physiology that has been observed empirically for L5PTs (Table S2). Subsequently, we refer to models that are consistent with these empirical observations as 'acceptable' (Fig. S1c).

Exploration of the biophysical parameter space generally failed to generate acceptable models for any of the L5PT morphologies (Fig. 1a), particularly for the generation of calcium APs and coincidence detection (Fig. 1b). We therefore considered that some important parameters that are necessary to capture the complex dendritic physiology of L5PTs might be missing in the models. We found one study, which reported that the expression of slow inactivating potassium channels steeply decays with soma distance along the apical dendrite of L5PTs ${ }^{27}$. The functional relevance of this observation remained, however, unclear to this date. Remarkably, when we incorporated parameters for the slope and offset of such potassium channels (Methods), exploration of the hence extended parameter space resulted in acceptable models. In fact, additional explorations continued to produce new sets of models with unique biophysical parameter combinations - i.e., with different channel distributions and densities, and passive electrical properties. Thus, as it increases the likelihood to generate acceptable models, a decay of slow inactivating potassium channels along the apical dendrite appears to be key for the robust function of L5PTs, particularly for generating calcium APs and coincidence detection. 

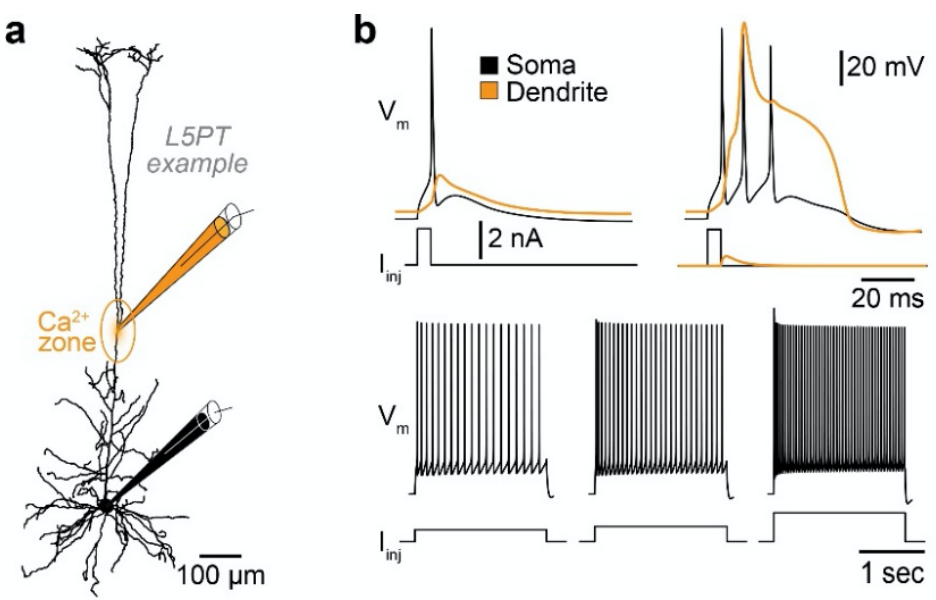

C

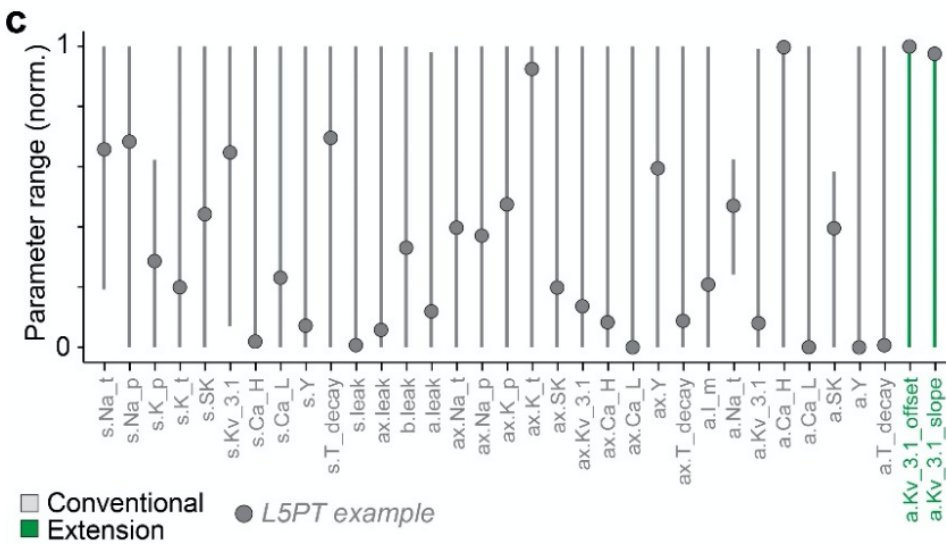

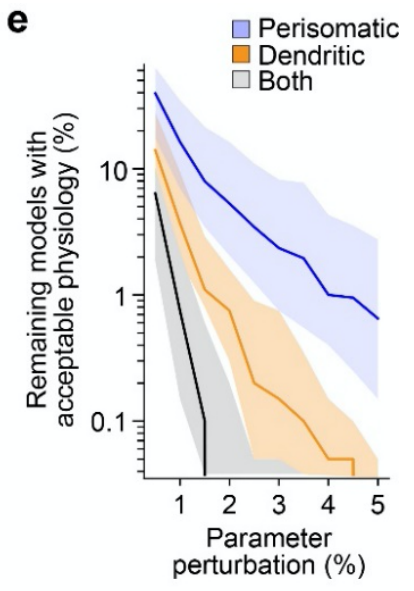

d

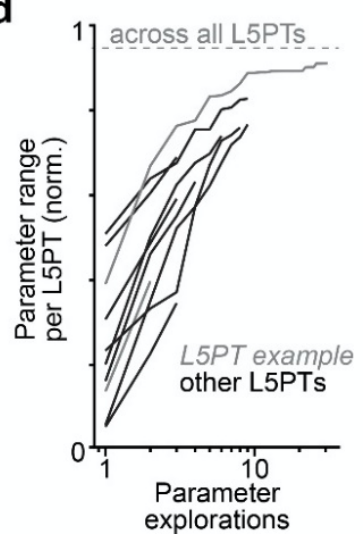

Fig. 1: Generating functionally realistic models for in vivo-labeled cortical neurons. a. Example dendrite morphology from the 12 layer 5 pyramidal tract neurons (L5PTs) for which we generated multi-compartmental models (Fig. S1a). The main branch point of the apical dendrite reflects the initiation zone for calcium $\left(\mathrm{Ca}^{2+}\right)$ action potentials (APs). b. Example simulations of current injections into the soma (black) and/or $\mathrm{Ca}^{2+}$ zone (orange) of a model for the L5PT from panel a. Consistent with empirical recording data (Table S2), all models analyzed here support the characteristic dendritic (top) and perisomatic (bottom) physiology of L5PTs (Fig. S1c), including backpropagation of APs into the 110 apical dendrite (top-left), calcium APs and burst firing when inputs to the soma and $\mathrm{Ca}^{2+}$ zone coincide (top-right), and regular AP firing of increasing frequencies in response to sustained current injections of increasing amplitude (bottom). c. Range of biophysical parameters across all models with acceptable dendritic and perisomatic physiology ( $\mathrm{n}=4.2$ millions) representing the passive leak conductance and the density of Hodgkin-Huxley type ion channels on the soma (s), basal dendrite (b), apical dendrite 115 (a), and axon initial segment (ax). We extended the parametrization of ion channel distributions reported by Hay et al., ${ }^{13}$ by two parameters (green) that allows the density of the fast non-inactivating potassium channel (Kv3.1) to decay along the apical dendrite (slope) until a minimum is reached (offset) ${ }^{27}$. Parameters are normalized to their biophysically plausible range (Methods). Dots represent the example model from panel b. d. Each exploration of the parameter space leads to new acceptable models that extend the parameter range for each morphology. Across all morphologies (dashed line), $95 \%$ of the plausible parameter ranges are covered (i.e., mean range across all parameters across all models). The parameter ranges covered by individual morphologies approach that across all morphologies with increasing numbers of parameter space explorations. e. Perturbing the parameters of acceptable models results in physiology that is no longer within the empirically observed range. Dendritic physiology is more sensitive to perturbations than perisomatic physiology. 'Remaining models' refers to the fraction of models that were acceptable out of randomly sampled models from the parameter space where each parameter was perturbed by $\leq 1 \%, 2 \%$, etc. 
We generated a large set of acceptable models $(n=4.2$ million $)$ to map out the spectrum of biophysical parameters by which the morphologically diverse sample of L5PTs could implement their functions. Surprisingly, we found that only very few parameters are strongly constrained. Instead, most parameters ranged from the minimal plausible value to the maximal one (Fig. 1c). The wide spectrum of parameters could simply reflect morphological variability. To address this possibility, we computed the parameter range across all models for each individual morphology. Even for the same morphology, the biophysical parameters covered up to $92 \%$ of the plausible range (Fig. 1d), thereby rivaling the parameter range observed across all morphologies combined (95\%). Does this mean that L5PTs are insensitive to changes in the distributions and densities of particular ion channels? To address this possibility, we perturbed the parameters of acceptable models. Perturbations of $1 \%$ in all parameters substantially changed the resulting intrinsic physiology, so that $>99 \%$ of the models were no longer acceptable (Fig. 1e). Thus, while the spectrum of channel expression patterns by which the different 140 L5PTs could implement their functions is equally widespread, each of the possible implementations within this spectrum is highly specific for the respective morphology.
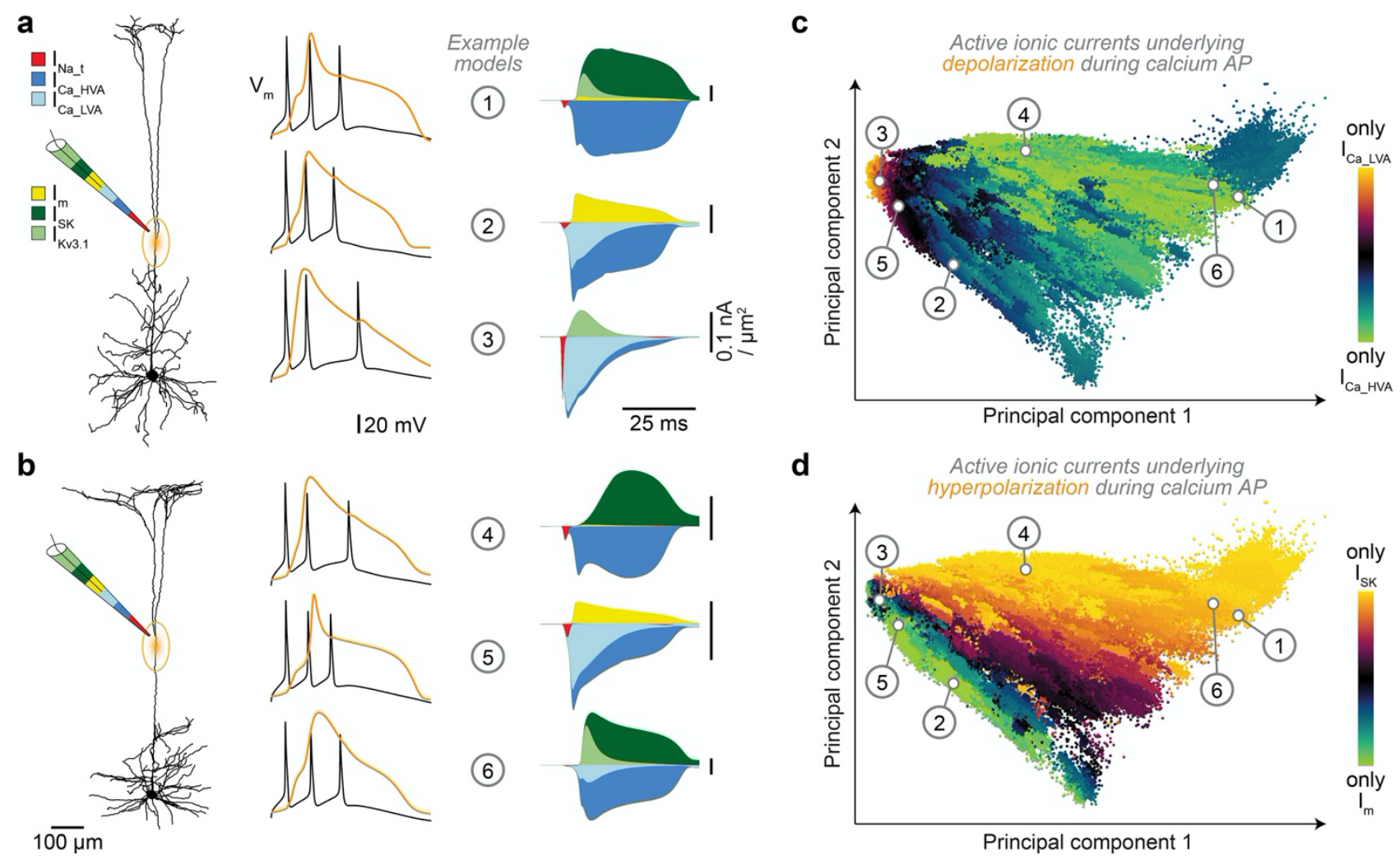

145 Fig. 2: Active computations in cortical dendrites arise from a wide spectrum of ionic currents. a. Three example models of the same morphology (left) that generate $\mathrm{Ca}^{2+}$ APs and axonal bursts of APs during coincidence detection within the experimentally observed range (middle), but which utilize very different superpositions of ion channels to achieve this function (right). Hyperpolarizing currents are plotted upwards (i.e., calcium-dependent (SK), fast non-inactivating (Kv3.1), and muscarinic (M) 150 potassium channels), depolarizing currents downwards (i.e., low- and high-voltage activated calcium channels (Ca_LVA and $\mathrm{Ca} \_\mathrm{HVA}$ ) and sodium ( $\mathrm{Na}$ - $\mathrm{t}$ ) channels). b: Example models with diverse channel unitizations similar to those in panel a, but for a different L5PT morphology. c. Principal component space (Methods) illustrating the wide spectrum of active ionic currents that can theoretically underlie dendritic calcium APs, color-coded by the contribution of the two most strongly utilized depolarizing channels. Visualized are 15.5 million models that capture the characteristic dendritic physiology of L5PTs, and which include the 4.2 million models that additionally capture the characteristic perisomatic physiology (Fig. S3). d. Same as in panel c, but color-coded for the two channels with the strongest contribution to hyperpolarization. 
Next, we investigated how L5PTs dynamically utilize ion channels - i.e., how their opening and/or closing contributes to the currents flowing in and out of the soma, axon and dendrites. For this purpose, we focused on the generation of calcium APs during coincidence detection, as these mechanisms require proper channel expression patterns that capture both the perisomatic and dendritic physiology (Fig. S2). For each of the acceptable models, we quantified the respective charge exchanged through the ion channels that are expressed at the initiation zone for calcium APs - i.e., around the main branch point of the apical dendrite (Fig. 2a). Although this was not an objective of the genetic algorithm, all models first used sodium and then calcium channels for depolarization, giving rise to $\mathrm{a} \mathrm{Na}^{+} / \mathrm{Ca}^{2+}$ complex as observed empirically ${ }^{28}$. Surprisingly, each model utilized the sodium, calcium and potassium channels very differently to generate calcium APs, even though the resulting shapes of the calcium APs and axonal AP bursts were very similar (Fig. 2a), and well within the experimentally observed range (Table S2). For example, some models relied entirely on high-voltage activated calcium channels (Ca_HVA) for depolarization, and used a superposition of calcium-dependent (SK) and fast non-inactivating potassium channels (Kv3.1) for hyperpolarization. Other models relied on low-voltage activated calcium channels (Ca_LVA) for depolarization, while using either the muscarinic (M) or Kv3.1 potassium channel for hyperpolarization. These observations generalized across morphologies (Fig. 2b). Thus, largely disparate channels utilizations can implement the same functions in L5PTs.

\begin{tabular}{r|lll|lll} 
& \multicolumn{3}{|c|}{$\begin{array}{c}\text { channel expression @BP } \\
\text { max conductance }(\mathrm{nA} / \mu \mathrm{m})\end{array}$} & \multicolumn{3}{c}{$\begin{array}{c}\text { channel utilization @BP } \\
\text { current contribution (\%) }\end{array}$} \\
\hline depolarization & model 1 & model 2 & model 3 & model 1 & model 2 & model 3 \\
\hline Ca_HVA & 49.83 & $\mathbf{3 5 . 0 3}$ & 3.22 & 99.12 & $\mathbf{6 9 . 6 9}$ & 19.92 \\
Ca_LVA & 1.49 & $\mathbf{1 8 8 5 . 3 3}$ & 834.80 & 0.03 & $\mathbf{2 9 . 3 2}$ & 74.97 \\
Na_t & 188.06 & 165.44 & 200.17 & 0.85 & 0.99 & 5.11 \\
hyperpolarization & model 1 & model 2 & model 3 & model 1 & model 2 & model 3 \\
\hline SK & 39.56 & 0.00 & 0.02 & 83.75 & 0.01 & 0.87 \\
M & 2.09 & 8.77 & 0.00 & 5.49 & 99.98 & 0.01 \\
Kv3.1 & $\mathbf{3 2 . 0 8}$ & 0.00 & $\mathbf{8 . 3 4}$ & $\mathbf{1 0 . 7 6}$ & 0.01 & $\mathbf{9 9 . 1 2}$
\end{tabular}

Table 1: Weak link between ion channel expression and utilization. We define ion channel utilization as the fraction of the charge exchanged through the specific channel in comparison to the charge exchanged through all other depolarizing or hyperpolarizing channels during a dendritic calcium AP. The relationship between ion channel expression and channel utilization is complex. First, comparing the expression of two ion channels in the same model illustrates that the channel that is expressed more is not necessarily utilized more. For example, model 2 expresses the low-voltage activated calcium (Ca_LVA) channel with a maximum conductance 54 times higher than that of the high-voltage activated calcium (Ca_HVA) channel, but the latter contributes the majority (69\%) of the depolarizing currents during the $\overline{\mathrm{Ca}}^{2+}$ AP. Second, comparing the expression of the same channel between two different models illustrates that the model that expresses a particular channel more does not necessarily rely more on it to perform its function. For example, model 1 expresses 4 times more of the fast non-inactivating potassium ( $\mathrm{Kv} 3.1$ ) channel compared to model 3, but this channel contributes $11 \%$ to hyperpolarization in model 1 versus $99 \%$ in model 3.

To analyze channel utilization more systematically, we represented each of the millions of models in a six-dimensional space, where each dimension reflects the charge exchanged through one of the channels that contribute to depolarization or hyperpolarization during calcium APs (Methods). The first two principal components of this space captured $>90 \%$ of the variability in channel utilization. While the utilization scenarios described above were located at the boundaries of the principle 
component space (Fig. 2c), the entire space was densely filled with models from the different morphologies. This indicates that the example scenarios reflect rather extreme cases of a wide spectrum by which L5PTs can utilize superpositions of different ion channels to implement the same function (Fig. 2d). Therefore, we considered that the wide spectrum of channel utilization could reflect the wide spectrum of channel expression that we observed across models. However, the more a particular channel was expressed did not necessarily imply that it was utilized more (Table 1). For example, the expression of low-voltage activated calcium channels might be much higher than that of the high-voltage activated ones, but the latter could still contribute the most to the depolarizing currents during the initiation of calcium APs. Similarly, models that expressed a particular channel more than others did not necessarily rely on it more to generate calcium APs. Thus, channel expression does not directly translate into how L5PTs utilize channels to implement their functions.

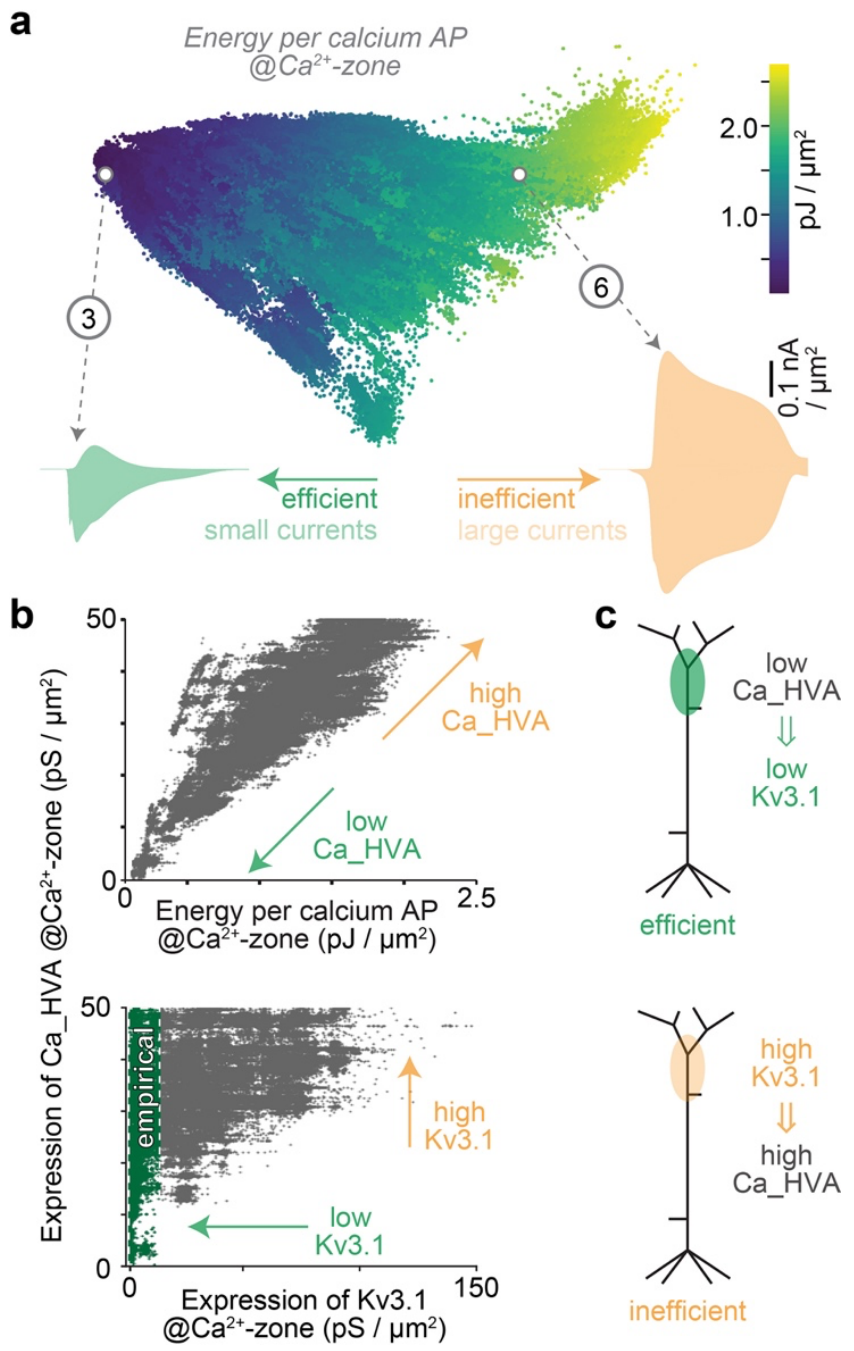

Fig. 3: Energy efficient signal processing constrains ion channels expression in cortical dendrites. a. Same principal component space as in Fig. 2c-d, but color-coded for the energy cost (Methods) of generating $\mathrm{Ca}^{2+}$ APs. Energy cost increases 10-fold along the first principal component of the theoretically possible channel utilization space. b. Top: Energy cost for calcium APs strongly correlates $(\mathrm{R}=0.94, \mathrm{p}<0.0001)$ with the expression of Ca_HVA channels in the distal apical dendrite (i.e., at the $\mathrm{Ca}^{2+}$ zone around the main branch point). Bottom: The expression of Ca_HVA channels restricts the expression of $\mathrm{Kv} 3.1$ channels. The empirically observed expression for slow inactivating potassium channels $^{27}$, which include Kv3.1 channels, is highlighted in green. c. Panel b reveals that energy efficient models are those that generate $\mathrm{Ca}^{2+}$ APs with low expression of Ca HVA, that a low expression of Kv3.1 channels is a prerequisite for a low expression of $\mathrm{Ca}$ HVA channels, and hence that efficient models require a low expression of Kv3.1 channels. Thus, because models with high expression of Kv3.1 channels in the distal dendrites are inconsistent with the empirical data $^{27}$, our findings indicate that L5PTs do not utilize these theoretically possible, but energy inefficient implementations of dendritic $\mathrm{Ca}^{2+}$ APs.

If neither diversity in morphology nor channel expression provide strong constraints for how L5PTs could implement their functions, which organizing principles underlie the wide spectrum of channel utilization? We noticed large differences in the current amplitudes that the different models required to generate calcium APs. Therefore, we computed the energy cost for the generation of calcium APs across all models (Methods). Energy cost correlated strongly (Pearson $R=0.94, p<0.0001$ ) with the first principle component of the channel utilization space (Fig. 3a). This remarkable relationship was independent of the perisomatic physiology (Fig. S3), and did not reflect a simple upscaling of channel activities in the distal dendrites. More specifically, if depolarizing and hyperpolarizing currents were scaled by a similar factor, the utilization of ion channels would essentially be the same, while energy 
costs would differ. However, the principal component space reflects gradual changes in the relative contribution of different depolarizing and hyperpolarizing currents. For example, models where SK channels dominate hyperpolarization gradually transition along the second principle component into models dominated by $\mathrm{M}$ channels, while all of these models consume similar energy to generate calcium APs. Thus, irrespective of energy cost, L5PTs could implement their functions with a wide spectrum of channel utilizations, which cannot be explained by similar scaling of channel activities.

255 Given that even the most energy-efficient implementation of a calcium AP could originate from a wide spectrum of channel expression and utilization, how will it be possible to test empirically whether the biophysical mechanisms in L5PTs are optimized for energy-efficient signal processing? For this purpose, we investigated whether particular expression patterns of ion channels are a prerequisite for low energy cost. Surprisingly, we found that energy cost correlated strongly $(R=0.94, p<0.0001)$ with the expression of high-voltage activated calcium channels (Fig. 3b). The higher the density of this channel at the initiation zone of calcium APs, the higher the energy cost. Furthermore, the expression of these calcium channels restricts the expression of the fast non-inactivating potassium channels (Fig. 3b). Specifically, energy efficient models need low expression of both high-voltage activated calcium and fast non-inactivating potassium channels. Vice versa, if the expression of fast non-inactivating potassium channels is high, L5PTs need a high expression of the calcium channels to generate calcium APs, which results in higher energy cost. Hence, if energy were to constrain the implementation of signal processing in L5PTs, the density of fast non-inactivating potassium channels needs to be low in the distal apical dendrite (Fig. 3c). This prediction is remarkably consistent with the empirical observations from the study that we used to extend the biophysical parameter space ${ }^{27}$. Thus, the steep decay of slow inactivating potassium channels does not only ensure robust function of L5PTs by facilitating coincidence detection, a low expression of such channels in the distal apical dendrites is also the prerequisite for an energy-efficient implementation of the underlying calcium AP.

\section{Discussion}

275 In this study we sought to reveal principles that govern relationships between morphology, channel expression and function in cortical neurons. By exploring an extended biophysical parameters space for a representative sample of in vivo labeled morphologies, we found that L5PTs could choose from a wide spectrum of both ion channel expressions and utilizations to implement their characteristic perisomatic and dendritic functions. Surprisingly, morphology only weakly constrains this spectrum i.e., channel expressions and utilizations are as diverse for individual morphologies as they are across all morphologies. Thus, independent of morphology, each L5PT has an enormous degree of freedom to perform its functions through which it might access the whole spectrum of channel utilizations that could account for active dendritic computations. Thereby, contrary to conventional views ${ }^{29}$, morphological variability is not the primary source for variability in channel expression. Beyond 285 compensating morphological differences, the degrees of freedom for channel expression remain extremely large. This finding indicates that variability in one scale - morphology, channel expression, channel utilization, and function - can only account for a minority of the variability in the other scales.

Previous work had demonstrated that similar neuronal function might arise from largely disparate implementations. For example, experimental ${ }^{30,31}$ and computational ${ }^{31-33}$ studies of the pyloric rhythm in the stomatogastric nervous system of cancer borealis found that different channel densities give rise to virtually indistinguishable activity patterns. Furthermore, multi-compartmental models of the same Purkinje cell morphology reproduced experimental activity data despite different channel expression patterns ${ }^{34}$. Supporting these previous observations, we demonstrate that also for active mechanisms in 295 the dendrites of cortical neurons, cellular properties do not need to be regulated to specific values to allow for proper function. However, we found that the energy cost for the generation of calcium APs organizes the spectrum of possible implementations. Energy-efficient implementations impose strict 
requirements on dendritic ion channel expression patterns, which are consistent with those observed empirically. We therefore propose that cortical neurons do not utilize all possible ways to implement their functions, but instead select those optimized for energy-efficient active dendritic computations.

Recent findings have linked calcium APs in L5PTs of the rodent primary somatosensory cortex to perception $^{35,36}$ and consciousness ${ }^{37,38}$. Thus, our findings might represent a strategy that allowed the cerebral cortex to evolve towards energy-efficient implementations of higher brain functions.

\section{Materials and Methods}

All relevant data and codes are available from the authors. Representative models for each morphology and the simulation routines, including a documentation of all parameters and the analysis routines can be obtained from ModelDB (https://senselab.med.yale.edu/ModelDB/; accession number: 267217).

Multi-compartmental models: We generated and analyzed 15.5 million multi-compartmental models for dendritic morphologies that represent 12 L5PTs from the rat barrel cortex (Fig. S1a). The morphologies originated from studies ${ }^{24,26,39}$ in which we had labeled individual neurons in vivo with Biocytin via cell-attached recordings (Wistar rats, postnatal day 25-46, male/female, Charles River). In these studies, we reported dendrite reconstructions for 91 excitatory neurons whose somata were located in the infragranular layers 5/6. Based on soma depth and 21 morphological features ${ }^{25}$, these neurons were objectively identified as L5PTs $(n=44)$, or as one of the other major excitatory cell types in layers 5/6 $(n=47)$ - i.e., intratelencephalic (L5IT), corticocortical (L6CC) or corticothalamic (L6CT) neurons ${ }^{40}$. From these data, we selected 12 L5PTs that were representative for the range of soma depth locations and dendritic features (e.g. path length, branch points, distance between the soma and the main branch point of the apical dendrite) observed across all reconstructed neurons of this cell type (Fig. S1b). The soma-dendrite reconstructions of the 12 L5PTs were converted into multicompartmental models. First, a simplified axon morphology was attached to the soma as described previously ${ }^{22}$. The axon consisted of an axon hillock with a diameter tapering from $3 \mu \mathrm{m}$ to $1.75 \mu \mathrm{m}$ over a length of $20 \mu \mathrm{m}$, an axon initial segment (AIS) of $30 \mu \mathrm{m}$ length and diameter tapering from 1.75 $\mu \mathrm{m}$ to $1 \mu \mathrm{m}$ diameter, and $1 \mathrm{~mm}$ of myelinated axon (diameter of $1 \mu \mathrm{m}$ ). Second, spatial discretization of the dendrite morphology (i.e., compartmentalization) was performed by computing the electrotonic length constant of each dendrite branch at a frequency of $100 \mathrm{~Hz}$ and setting the length of individual compartments in this branch to $10 \%$ of this length constant. Third, we incorporated the dynamical models of ion channels and intracellular calcium dynamics developed by Hay et al., ${ }^{13}$ and assigned them to the soma, basal dendrites, apical dendrite and/or AIS, as summarized in Table S1. The resulting biophysical parameters were the passive leak conductance, the maximum conductance of each ion channel, and two parameters characterizing the calcium dynamics in the soma, axon, basal and apical dendrites. In addition to these free parameters, the models include the non-specific cation 335 current $\mathrm{I}_{\mathrm{h}}$, which increases exponentially with soma distance to match the empirically observed voltage slope along the dendritic tree ${ }^{13}$. Calcium channels on the apical dendrite were expressed in high density for $\pm 100 \mu \mathrm{m}$ around the main branch point. Outside the hence defined calcium zone, and for oblique dendrites in general, calcium channels along the apical dendrite were expressed with $1 / 100^{\text {th }}$ and $1 / 10^{\text {th }}$ of the calcium zone density for Ca_LVA and Ca_HVA, respectively. To account for the empirically observed decay along the apical dendrite of slow inactivating potassium channels ${ }^{27}$, we defined a slope parameter for the linear decrease of the density of fast non-inactivating potassium (Kv3.1) channels with soma distance, and an off set parameter for the minimum density of Kv3.1 channels throughout the apical dendrite. Kv3.1 channel density on the apical dendrite is hence defined as follows:

$$
g(l)=\max \left(\bar{g} \cdot \text { offset }, \bar{g} \cdot\left(1-\operatorname{slope} \cdot \frac{l}{L}\right)\right),
$$


where $l$ denotes the path length to the soma, $g(l)$ the maximum conductance of the Kv3.1 channel on the apical dendrite as a function of path length to the soma, $\bar{g}$ the maximum conductance of the Kv3.1 channel on the apical dendrite closest to the soma, and $L$ the maximum path length (i.e., the distance of the dendritic segment with the largest distance to the soma). Furthermore, we added a parameter to scale the diameters of apical dendrites, which were reconstructed at the resolution limit of light microscopy. The resulting range for the diameters of the apical dendrite (ranging from 1.3 to 4.2 at the main branch point) was consistent with empirical measurements in rat somatosensory ${ }^{41}$ and visual ${ }^{42}$ cortex. The average diameter at the main branch point of the apical dendrite was $3.1 \mu \mathrm{m}$, resembling measurements at electron microscopic resolution for layer 5 pyramidal neurons in mouse barrel cortex $^{43}$. The total number of free parameters in each model was hence 35 (i.e., 32 from the conventional model reported by the Hay et al., ${ }^{13}, 2$ for the decay of the Kv3.1 channel along the apical dendrite, and one for scaling its diameter).

Simulations: Simulations of multi-compartmental models were performed with NEURON $7.4^{44}$ and custom written software in python. Temperature was set to $34^{\circ} \mathrm{C}$, and the models where initialized with a membrane potential of $-75 \mathrm{mV}$. The biophysical parameters (e.g. distributions of ion channels, intracellular calcium dynamic), and the diameters of the apical dendrite were optimized using the multi-objective evolutionary algorithm developed by Hay et al., ${ }^{13}$ until simulated responses to current injections reproduced the characteristic responses of L5PTs within the empirically observed range

365 (Table S2). For optimization, the parameters were normalized with respect to the biophysically plausible ranges as reported by Hay et al., ${ }^{13}$. For the 3 additional parameters, we used the following ranges: 0.5 to 3 for scaling of apical dendrite, 0 to 1 and -3 to 0 for the offset and slope of the Kv.3.1 channel density, respectively. Optimization was performed to capture the following dendritic physiology of L5PTs (Fig. S2): backpropagating APs after a brief somatic current injection (1.9 nA, 5 $\mathrm{ms})$, and $\mathrm{Ca}^{2+}$ APs during coincidence detection after a brief somatic current injection (1.9 nA, $5 \mathrm{~ms}$ ) followed by an EPSP like current injection ( $5 \mathrm{~ms}$ delay, $0.5 \mathrm{nA}$ ) $180 \mu \mathrm{m}$ below to the main branch point of the apical dendrite. These current injections were applied $295 \mathrm{~ms}$ after initialization of the simulation. To capture the characteristic perisomatic physiology of L5PTs, parameter optimization was additionally performed for somatic step current injections with the $0.619 \mathrm{nA}, 0.793 \mathrm{nA}$ and 1.507

$375 \mathrm{nA}$ amplitudes and a duration of 2 seconds. These current injections were applied $700 \mathrm{~ms}$ after initialization of the simulation. During the optimization, the 40 features of the simulated voltage traces were compared to the respective empirical data as proposed by Hay et al., ${ }^{13}$, and as detailed in Table S2. The features were normalized by z-scoring with respect to the experimentally observed mean and standard deviation. Models were considered acceptable if the maximum z-scored deviation was less than 3.1 for the dendritic physiology, and less than 4.5 for the perisomatic physiology. The mean deviation of the thereby selected models was $1.05 \pm 0.14$ (Fig. S1), well below the cutoff criterion for acceptable models that was suggested previously ${ }^{45}$. We used the indicator based evolutionary (IBEA) algorithm $^{22}$ for optimization with a population size of 1000 , mutation probability of 0.3 , and crossingover probability of 0.7 . We used dask to parallelize the optimization. The optimization was performed 385 in two steps. First, optimization was performed for 600 generations to capture the dendritic physiology. Second, if models with a maximum deviation of $<3$ standard deviations were found, we continued the optimization by incorporating the perisomatic physiology into the objectives. The optimization was terminated if there was no progress, or when acceptable models were found. Repeating the optimization procedure with different seeds generally resulted in new models, which extended the parameter ranges for each morphology. We continued this parameter space exploration until the parameter range coverage was close to $100 \%$, and the principal component space was densely filled.

Quantification and statistical analysis: For all models with acceptable dendritic physiology (i.e., including those with acceptable perisomatic physiology), we extracted the membrane potential, currents through active ion channels, and the intracellular calcium concentration at the soma and main 
branch point of the apical dendrite during simulations of coincidence detection. From these data, we computed the total charge exchanged (unit: Coulomb) through each of the seven ion channels that are expressed at the main branch point by integrating the currents for a $50 \mathrm{~ms}$ time interval starting with the onset of the somatic current injection. Currents through $\mathrm{I}_{h}$ channels were negligible in comparison to the other six ionic currents, and discarded from further analysis (the density $\mathrm{I}_{\mathrm{h}}$ channels was also not subject to optimization). The charge exchanged through the remaining six ion channels was then used to compute the first two principal components across all acceptable models. Among the three depolarizing channels - voltage-gated sodium channel, high- and low-voltage activated calcium channels - the latter were dominating the generation of $\mathrm{Ca}^{2+}$ APs during coincidence detection across all models. Thus, we computed the depolarization index in Fig. 2c only for the calcium channels:

$$
\frac{\text { charge exchanged }\left(\mathrm{Ca}_{H V A}\right)-\text { charge exchanged }\left(\mathrm{Ca}_{L V A}\right)}{\text { charge exchanged }\left(\mathrm{Ca}_{H V A}\right)+\text { charge exchanged }\left(\mathrm{Ca}_{L V A}\right)}
$$

Similarly, M and SK channels dominated hyperpolarization across the majority of models, and we 410 hence computed the hyperpolarization index in Fig. 2d only for these two potassium channels. To estimate the energy cost of dendritic $\mathrm{Ca}^{2+}$ APs, we computed the difference between membrane potential and the reversal potential of each ionic species (potassium: $-85 \mathrm{mV}$, sodium: $50 \mathrm{mV}$, calcium: the reversal potential was constantly updated based on the varying intracellular $\mathrm{Ca}^{2+}$ concentration using the Nernst equation). These potential differences were multiplied with the respective ionic currents and integrated over the $50 \mathrm{~ms}$ time interval using Simpson's rule. Statistical tests were performed using the scipy python package (version 0.18.2).

\section{References}

1. Sterling, P. \& Laughlin, S. Principles of Neural Design (The MIT Press, Cambridge, Massachusetts, 2015).

2. Yu, L. \& Yu, Y. Energy-efficient neural information processing in individual neurons and neuronal networks. J Neurosci Res 95, 2253-2266 (2017).

3. Bear, M.F., Connors, B.W. \& Paradiso, M.A. Neuroscience: Exploring the Brain (Williams \& Wilkins, Baltimore, 1996).

425 4. Harris, J.J. \& Attwell, D. The energetics of CNS white matter. J Neurosci 32, 356-371 (2012).

5. Howarth, C., Gleeson, P. \& Attwell, D. Updated energy budgets for neural computation in the neocortex and cerebellum. J Cereb Blood Flow Metab 32, 1222-1232 (2012).

6. Alle, H., Roth, A. \& Geiger, J.R. Energy-efficient action potentials in hippocampal mossy fibers. Science 325, 1405-1408 (2009).

430 7. Carter, B.C. \& Bean, B.P. Sodium entry during action potentials of mammalian neurons: incomplete inactivation and reduced metabolic efficiency in fast-spiking neurons. Neuron 64, 898-909 (2009).

8. Sengupta, B., Stemmler, M., Laughlin, S.B. \& Niven, J.E. Action potential energy efficiency varies among neuron types in vertebrates and invertebrates. PLoS Comput Biol 6, e1000840 (2010).

9. Hodgkin, A. The optimum density of sodium channels in an unmyelinated nerve. Philos Trans $R$ Soc Lond B Biol Sci 270, 297-300 (1975).

10. Hodgkin, A.L. \& Huxley, A.F. Movement of sodium and potassium ions during nervous activity. Cold Spring Harb Symp Quant Biol 17, 43-52 (1952).

440 11. Schmidt-Hieber, C. \& Bischofberger, J. Fast sodium channel gating supports localized and efficient axonal action potential initiation. J Neurosci 30, 10233-10242 (2010).

12. Hasenstaub, A., Otte, S., Callaway, E. \& Sejnowski, T.J. Metabolic cost as a unifying principle governing neuronal biophysics. Proc Natl Acad Sci U S A 107, 12329-12334 (2010). 
13. Hay, E., Hill, S., Schurmann, F., Markram, H. \& Segev, I. Models of neocortical layer 5b pyramidal cells capturing a wide range of dendritic and perisomatic active properties. PLoS Comput Biol 7, e1002107 (2011).

14. Ramaswamy, S. \& Markram, H. Anatomy and physiology of the thick-tufted layer 5 pyramidal neuron. Front Cell Neurosci 9, 233 (2015).

15. Kim, H.G. \& Connors, B.W. Apical dendrites of the neocortex: correlation between sodium- and calcium-dependent spiking and pyramidal cell morphology. J Neurosci 13, 5301-5311 (1993).

16. Magee, J., Hoffman, D., Colbert, C. \& Johnston, D. Electrical and calcium signaling in dendrites of hippocampal pyramidal neurons. Annu Rev Physiol 60, 327-346 (1998).

17. Schiller, J., Schiller, Y., Stuart, G. \& Sakmann, B. Calcium action potentials restricted to distal apical dendrites of rat neocortical pyramidal neurons. J Physiol 505 ( Pt 3), 605-616 (1997).

18. Spencer, W.A. \& Kandel, E.R. Electrophysiology of Hippocampal Neurons: Iv. Fast Prepotentials. J Neurophysiol 24, 272-285 (1961).

19. Larkum, M.E., Zhu, J.J. \& Sakmann, B. A new cellular mechanism for coupling inputs arriving at different cortical layers. Nature 398, 338-341 (1999).

20. Rapp, M., Yarom, Y. \& Segev, I. Modeling back propagating action potential in weakly excitable dendrites of neocortical pyramidal cells. Proc Natl Acad Sci U S A 93, 11985-11990 (1996).

21. Schaefer, A.T., Larkum, M.E., Sakmann, B. \& Roth, A. Coincidence detection in pyramidal neurons is tuned by their dendritic branching pattern. J Neurophysiol 89, 3143-3154 (2003).

22. Hay, E., Schurmann, F., Markram, H. \& Segev, I. Preserving axosomatic spiking features despite diverse dendritic morphology. J Neurophysiol 109, 2972-2981 (2013).

23. Marder, E. \& Goaillard, J.M. Variability, compensation and homeostasis in neuron and network function. Nat Rev Neurosci 7, 563-574 (2006).

24. Narayanan, R.T., et al. Beyond Columnar Organization: Cell Type- and Target Layer-Specific Principles of Horizontal Axon Projection Patterns in Rat Vibrissal Cortex. Cereb Cortex 25, 4450-4468 (2015).

25. Oberlaender, M., et al. Cell type-specific three-dimensional structure of thalamocortical circuits in a column of rat vibrissal cortex. Cereb Cortex 22, 2375-2391 (2012).

26. Rojas-Piloni, G., et al. Relationships between structure, in vivo function and long-range axonal target of cortical pyramidal tract neurons. Nat Commun 8, 870 (2017).

27. Schaefer, A.T., et al. Dendritic voltage-gated $\mathrm{K}+$ conductance gradient in pyramidal neurones of neocortical layer 5B from rats. $J$ Physiol 579, 737-752 (2007).

28. Larkum, M.E., Zhu, J.J. \& Sakmann, B. Dendritic mechanisms underlying the coupling of the dendritic with the axonal action potential initiation zone of adult rat layer 5 pyramidal neurons. $J$ Physiol 533, 447-466 (2001).

29. Mainen, Z.F. \& Sejnowski, T.J. Influence of dendritic structure on firing pattern in model neocortical neurons. Nature 382, 363-366 (1996).

30. Golowasch, J., Casey, M., Abbott, L.F. \& Marder, E. Network stability from activity-dependent regulation of neuronal conductances. Neural Comput 11, 1079-1096 (1999).

31. Golowasch, J., Goldman, M.S., Abbott, L.F. \& Marder, E. Failure of averaging in the construction of a conductance-based neuron model. J Neurophysiol 87, 1129-1131 (2002).

32. Goldman, M.S., Golowasch, J., Marder, E. \& Abbott, L.F. Global structure, robustness, and modulation of neuronal models. J Neurosci 21, 5229-5238 (2001).

33. Prinz, A.A., Bucher, D. \& Marder, E. Similar network activity from disparate circuit parameters. Nat Neurosci 7, 1345-1352 (2004).

34. Achard, P. \& De Schutter, E. Complex parameter landscape for a complex neuron model. PLoS Comput Biol 2, e94 (2006).

35. Takahashi, N., et al. Active dendritic currents gate descending cortical outputs in perception. Nat Neurosci 23, 1277-1285 (2020). 
36. Takahashi, N., Oertner, T.G., Hegemann, P. \& Larkum, M.E. Active cortical dendrites modulate perception. Science 354, 1587-1590 (2016).

37. Aru, J., Suzuki, M. \& Larkum, M.E. Cellular Mechanisms of Conscious Processing. Trends Cogn Sci 24, 814-825 (2020).

38. Suzuki, M. \& Larkum, M.E. General Anesthesia Decouples Cortical Pyramidal Neurons. Cell 180, 666-676 e613 (2020).

39. Guest, J.M., Bast, A., Narayanan, R.T. \& Oberlaender, M. Thalamus gates active dendritic computations in cortex during sensory processing. (bioRxiv, 2021).

40. Harris, K.D. \& Shepherd, G.M. The neocortical circuit: themes and variations. Nat Neurosci 18, 170-181 (2015).

41. de Kock, C.P., Bruno, R.M., Spors, H. \& Sakmann, B. Layer- and cell-type-specific suprathreshold stimulus representation in rat primary somatosensory cortex. J Physiol 581, 139154 (2007).

42. Larkman, A. \& Mason, A. Correlations between morphology and electrophysiology of pyramidal neurons in slices of rat visual cortex. I. Establishment of cell classes. J Neurosci 10, 1407-1414 (1990).

43. Karimi, A., Odenthal, J., Drawitsch, F., Boergens, K.M. \& Helmstaedter, M. Cell-type specific innervation of cortical pyramidal cells at their apical dendrites. Elife 9 (2020).

44. Hines, M.L. \& Carnevale, N.T. The NEURON simulation environment. Neural Comput 9, 11791209 (1997).

45. Gouwens, N.W., et al. Systematic generation of biophysically detailed models for diverse cortical neuron types. Nature Communications 9, 710 (2018).

\section{Acknowledgments}

We thank Bert Sakmann for comments on the manuscript, Etay Hay for providing the conventional biophysical model and genetic algorithm, and Christiaan de Kock for providing some of the morphologies. Funding was provided by the Center of Advanced European Studies and Research, the German Federal Ministry of Education and Research (grant 01IS18052, to M.O.), the Deutsche Forschungsgemeinschaft (SFB 1089 and SPP 2041, to M.O.), and the European Research Council under the European Union's Horizon 2020 research and innovation program (grant 633428, to M.O.). Author contributions: M.O. and A.B. conceived and designed the study. A.B. developed the model, performed simulations, and analyzed the data. M.O. and A.B. wrote the paper. The authors declare no competing interests. 

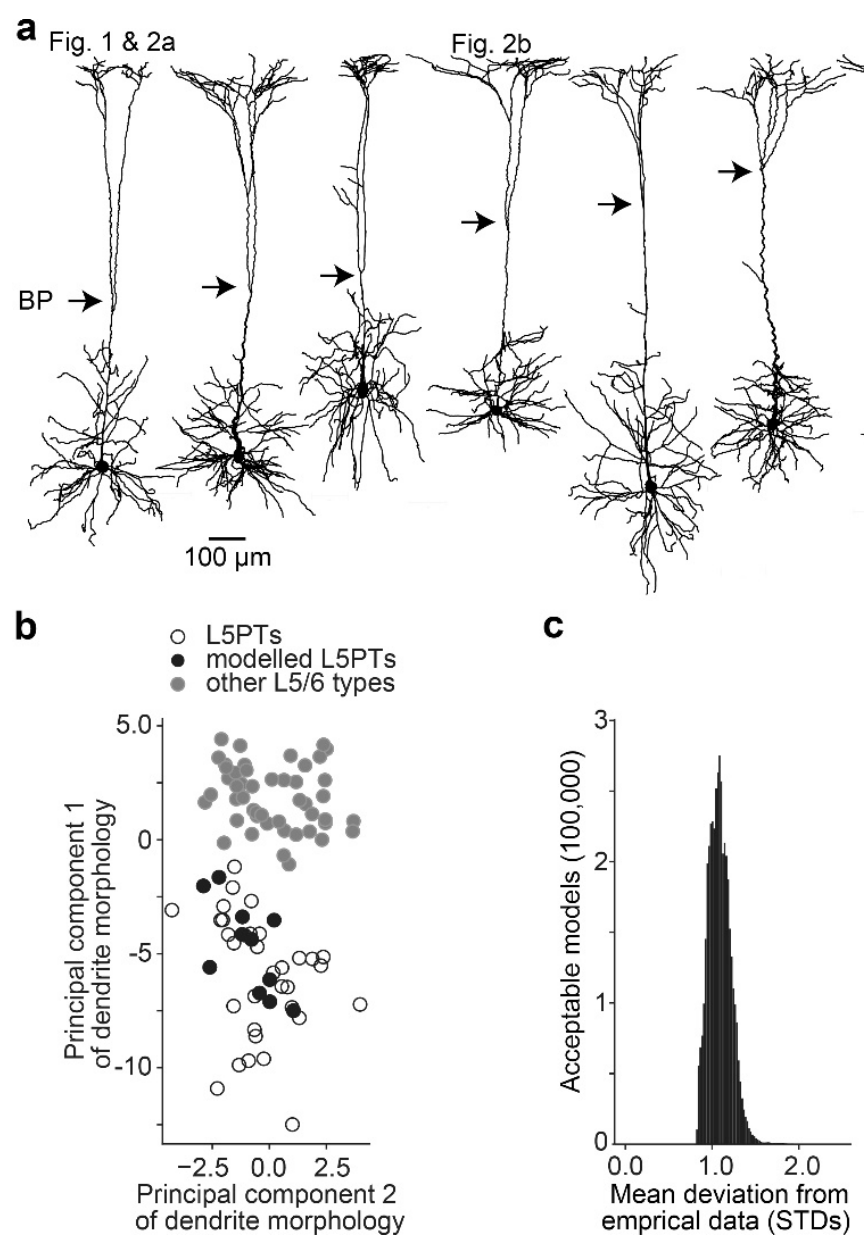

Fig. S1 (related to Fig. 1): Models represent the morphological diversity of L5PTs. a. Gallery of the in vivo-labeled dendrite morphologies used in this study. The main branch point (BP) of the apical dendrite reflects the initiation zone for $\mathrm{Ca}^{2+}$ APs. For one of these morphologies (most right), we could generate models that reproduce the dendritic physiology of L5PTs, but no models that reproduce both dendritic and perisomatic physiology. b. The morphologies for which we generated models represent the morphological diversity of L5PTs in the rat barrel cortex. Here we visualize the first two principal components derived from 22 morphological features that reliably discriminate L5PTs $(n=44)$ from the other major excitatory cell types in layers $5 / 6(n=47 \text {; i.e., L5ITs, L6CCs, L6CTs })^{24}$. c. Mean error across all models with acceptable dendritic and perisomatic physiology, quantified as the deviation from empirical mean in units of standard deviations across all 40 objectives (Table S2). 

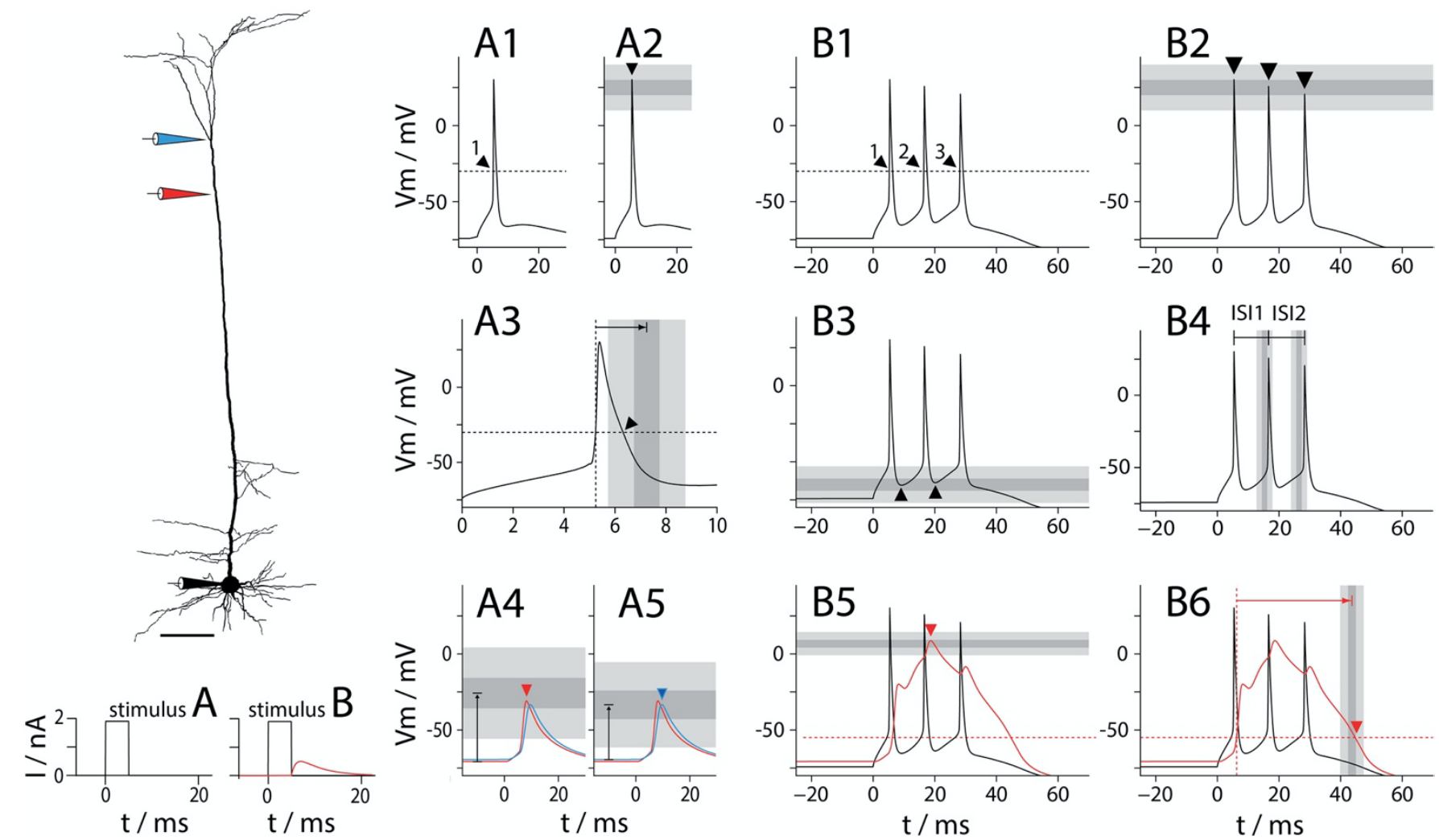

Fig. S2 (related to Fig. 2): Empirical constraints for models with acceptable dendritic physiology.

Panels A1-B6: features extracted from example simulations of responses to stimulus A (5 ms somatic step current injection of $1.9 \mathrm{nA}$ ) and stimulus B (as stimulus A, but combined with an EPSP like injection of $0.5 \mathrm{nA}$ into the $\mathrm{Ca}^{2+}$ zone of the apical dendrite). Grey corridors represent the mean of the empirical data \pm one standard deviation (dark grey), and three standard deviations (light grey) A1. Stimulus A evokes a single AP. A2. AP peak is $25 \pm 5 \mathrm{mV}$. A3. AP width is $2 \pm 0.5 \mathrm{~ms}$. A4. Amplitude of backpropagating AP at the red recording pipette (located $180 \mu \mathrm{m}$ below the main branch point) is $45 \pm 10 \mathrm{mV}$. A5. Amplitude at the blue recording pipette (located at the main branch point) is $36 \pm 9.33$ $\mathrm{mV}$. B1. Stimulus B evokes a burst of three axonal APs. B2. The mean AP peak across all 3 APs is $25 \pm 5 \mathrm{mV}$. B3. Mean somatic after-hyperpolarization (AHP) depth is $-65 \pm 4 \mathrm{mV}$. B4. The mean somatic inter-spike-interval (ISI) is $9.9 \pm 0.85 \mathrm{~ms}$. B5. The $\mathrm{Ca}^{2+}$ AP peak is $6.73 \pm 2.54 \mathrm{mV}$. B6. The $\mathrm{Ca}^{2+} \mathrm{AP}$ 
a

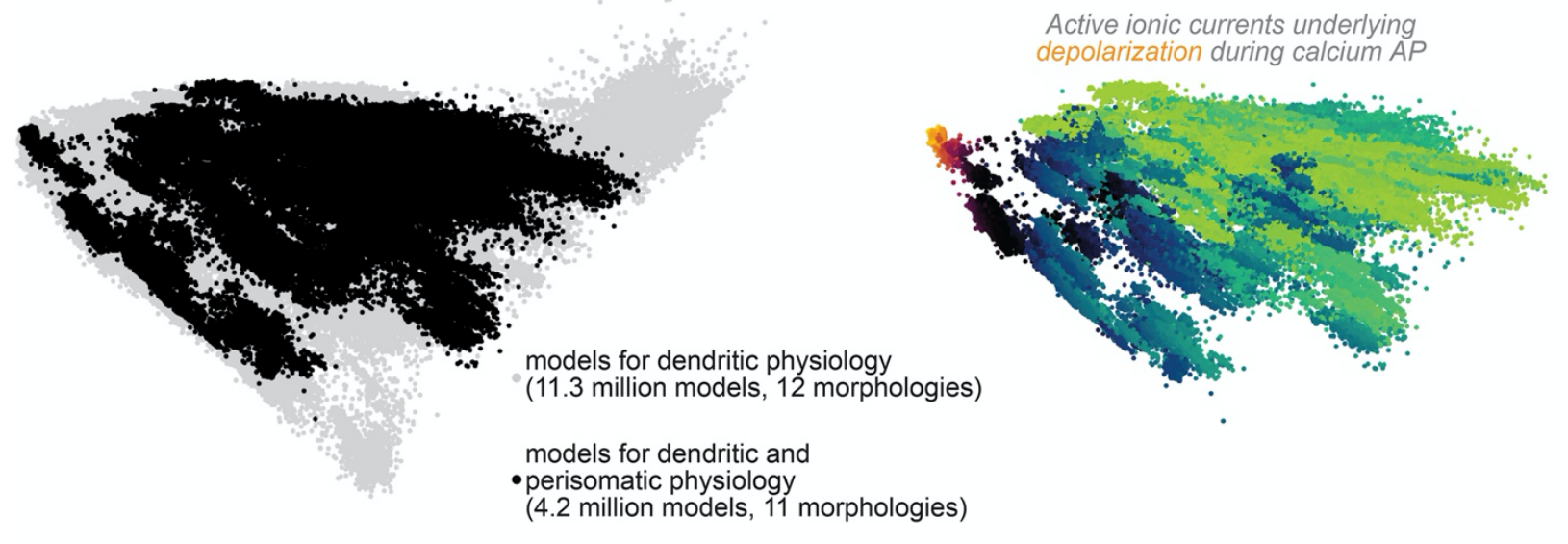

b

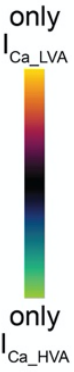

C

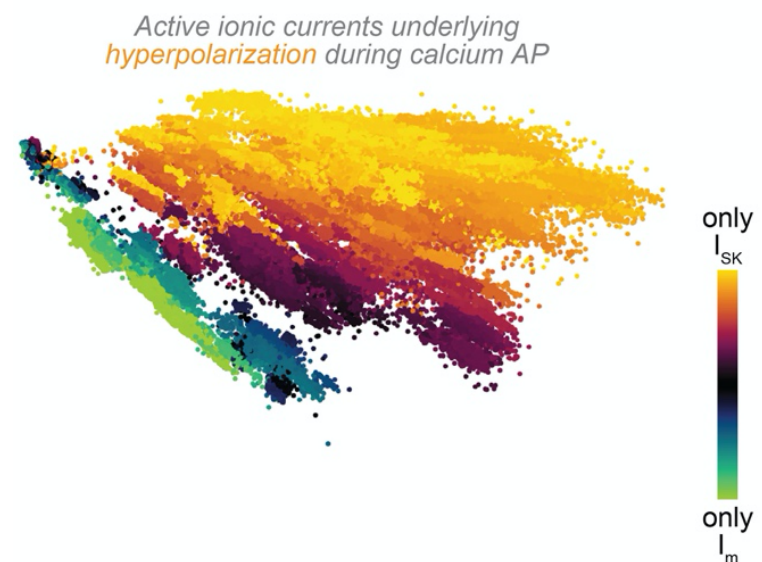

d

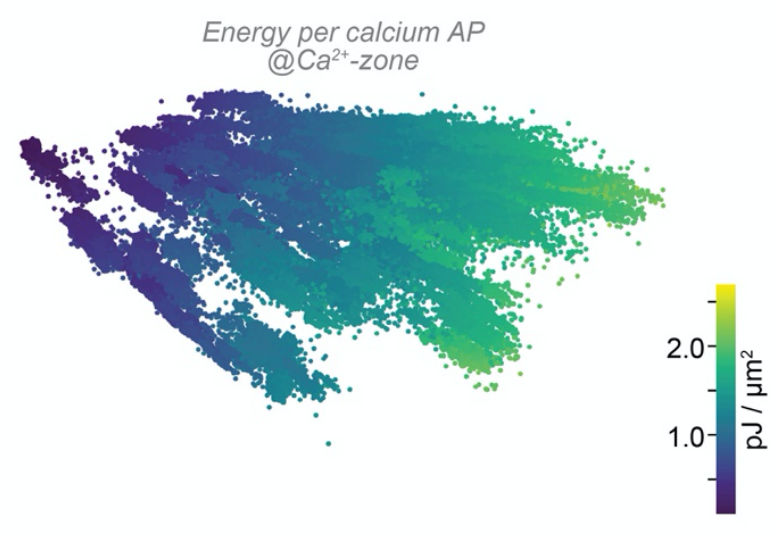

Fig. S3 (related to Fig. 3): Principles underlying the dendritic physiology of L5PTs are independent from the perisomatic physiology. a. Principle component space as in Fig. 2 and Fig. 3. Models which reproduce only the dendritic physiology of L5PTs ( $n=11.3$ million, grey dots) share the same space and intermingle with those models that capture both the dendritic and perisomatic physiology ( $n=4.2$ million, black dots). This indicates that perisomatic properties do not constrain the channel utilization required to capture the dendritic physiology. b-d. Same analysis as in Fig. 2 and Fig. 3, but restricted to the 4.2 million models that reproduce both the dendritic and perisomatic physiology. Consistent with Fig. 2c, models transition along principal component 1 from Ca_LVA as the predominant depolarizing mechanism to Ca_HVA (panel b). Consistent with Fig. 2d, models transition along principal component 2 from $\mathrm{M}$ currents as the dominating hyperpolarizing mechanism to calcium-dependent potassium currents (panel c). Consistent with Fig. 3a, the energy cost per $\mathrm{Ca}^{2+}$ AP increases along principal component 1 (panel d). Thus, the organizational principles underlying the generation of $\mathrm{Ca}^{2+}$ APs are largely unaffected by constraints that are required to capture the perisomatic physiology. 


\begin{tabular}{lcccc}
\hline & Soma & AIS & $\begin{array}{c}\text { Basal } \\
\text { Dendrites }\end{array}$ & $\begin{array}{c}\text { Apical } \\
\text { Dendrite }\end{array}$ \\
\hline$l e a k$ & $\mathrm{X}$ & $\mathrm{X}$ & $\mathrm{X}$ & $\mathrm{X}$ \\
$I_{h}$ & $\mathrm{X}$ & $\mathrm{X}$ & $\mathrm{X}$ & $\mathrm{X}$ \\
$I_{N a_{-} t}$ & $\mathrm{X}$ & $\mathrm{X}$ & & $\mathrm{X}$ \\
$I_{N a \_p}$ & $\mathrm{X}$ & $\mathrm{X}$ & & \\
$I_{K v 3.1}$ & $\mathrm{X}$ & $\mathrm{X}$ & & $\mathrm{X}$ \\
$I_{S K}$ & $\mathrm{X}$ & $\mathrm{X}$ & & $\mathrm{X}$ \\
$I_{K \_} p$ & $\mathrm{X}$ & $\mathrm{X}$ & & \\
$I_{K \_} t$ & $\mathrm{X}$ & $\mathrm{X}$ & & \\
$I_{m}$ & & & & $\mathrm{X}$ \\
$I_{C a_{-} H V A}$ & $\mathrm{X}$ & $\mathrm{X}$ & & $\mathrm{X}$ \\
$I_{C a_{-} L V A}$ & $\mathrm{X}$ & $\mathrm{X}$ & & $\mathrm{X}$ \\
$C a^{2+}-$ dynamics & $\mathrm{X}$ & $\mathrm{X}$ & & $\mathrm{X}$ \\
\hline & & & & \\
\hline
\end{tabular}

Table S1: Ionic currents by subcellular compartment. Abbreviations: Non-specific cation current, $\mathrm{I}_{\mathrm{h}}$. Fast inactivating $\mathrm{Na}^{+}$current, $\mathrm{I}_{\mathrm{Na} \_}$. Persistent $\mathrm{Na}^{+}$current, $\mathrm{I}_{\mathrm{Na} \_ \text {p }}$. Fast non-inactivating $\mathrm{K}^{+}$current, $\mathrm{I}_{\mathrm{Kv} \text { 3.1. }}$. Small-conductance $\mathrm{Ca}^{2+}$ activated $\mathrm{K}^{+}$current, $\mathrm{I}_{\mathrm{SK}}$. Slow inactivating $\mathrm{K}^{+}$current, $\mathrm{I}_{\mathrm{K} \_ \text {p }}$. Fast 570 inactivating $\mathrm{K}^{+}$current, $\mathrm{I}_{\mathrm{K}_{-}}$. High-voltage activated $\mathrm{Ca}^{2+}$ current, $\mathrm{I}_{\mathrm{Ca}-\mathrm{HVA}}$. Low-voltage activated $\mathrm{Ca}^{2+}$

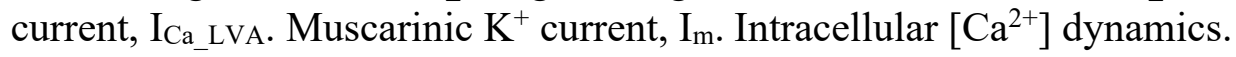




\begin{tabular}{|c|c|c|c|c|c|}
\hline \multicolumn{4}{|c|}{ Features of perisomatic physiology } & \multicolumn{2}{|l|}{ Features of dendritic physiology } \\
\hline Feature & current step 1 & $\begin{array}{l}\text { Mean } \pm \text { STD } \\
\text { current step } 2\end{array}$ & current step 3 & Feature & Mean \pm STD \\
\hline \multirow[t]{2}{*}{ 1. AP frequency $(\mathrm{Hz})$} & $9 \pm 0.88$ & $14.5 \pm 0.56$ & $22.5 \pm 2.22$ & 1. $\mathrm{Ca}^{2+} \mathrm{AP}$ peak $(\mathrm{mV})$ & $6.73 \pm 2.54$ \\
\hline & $9.44 \pm 0.83$ & $14.29 \pm 0.72$ & $24.57 \pm 1.83$ & & $7.36 \pm 1.92$ \\
\hline \multirow[t]{2}{*}{ 2. Adaptation index } & $0.0036 \pm 0.0091$ & $0.0023 \pm 0.0056$ & $0.0046 \pm 0.0026$ & 2. $\mathrm{Ca}^{2+} \mathrm{AP}$ width (mV) & $37.43 \pm 1.27$ \\
\hline & $0.0078 \pm 0.0088$ & $0.0071 \pm 0.0053$ & $0.0086 \pm 0.0035$ & & $37.63 \pm 0.96$ \\
\hline \multirow[t]{2}{*}{ 3. ISI-CV } & $0.1204 \pm 0.0321$ & $0.1083 \pm 0.0368$ & $0.0954 \pm 0.0140$ & \multirow{3}{*}{$\begin{array}{l}\text { 3. Somatic AP spike count } \\
\text { (coincident current injection) } \\
\text { 4. Somatic AP ISI (ms) }\end{array}$} & $3 \pm 0$ \\
\hline & $0.0866 \pm 0.0369$ & $0.0936 \pm 0.0281$ & $0.1165 \pm 0.0198$ & & $3 \pm 0$ \\
\hline \multirow[t]{2}{*}{ 4. Initial Burst ISI (ms) } & $57.75 \pm 33.48$ & $6.625 \pm 8.65$ & $5.38 \pm 0.83$ & & $9.9 \pm 0.85$ \\
\hline & $43.66 \pm 14.41$ & $17.17 \pm 5.62$ & $6.56 \pm 0.72$ & & $10.1 \pm 1.1$ \\
\hline \multirow[t]{2}{*}{ 5. First spike latency (ms) } & $43.25 \pm 7.32$ & $19.13 \pm 7.31$ & $7.25 \pm 1.00$ & 5. Somatic AHP depth (mV) & $-65 \pm 4$ \\
\hline & $34.38 \pm 5.41$ & $20.26 \pm 2.51$ & $7.01 \pm 0.89$ & & $-65 \pm 1$ \\
\hline \multirow[t]{2}{*}{ 6. AP peak (mV) } & $26.23 \pm 4.97$ & $16.52 \pm 6.11$ & $16.44 \pm 6.93$ & 6. Somatic AP peak (mV) & $25 \pm 5$ \\
\hline & $20.77 \pm 3.50$ & $20.58 \pm 3.56$ & $19.23 \pm 3.85$ & & $27 \pm 3$ \\
\hline \multirow[t]{2}{*}{ 7. Fast AHP depth (mV) } & $-51.95 \pm 5.82$ & $-54.19 \pm 5.57$ & $-56.56 \pm 3.58$ & 7. Somatic AP half-width (ms) & $2 \pm 0.5$ \\
\hline & $-61.68 \pm 1.17$ & $-61.53 \pm 1.21$ & $-60.52 \pm 1.43$ & & $0.99 \pm 0.05$ \\
\hline \multirow[t]{2}{*}{ 8. Slow AHP depth (mV) } & $-58.04 \pm 4.58$ & $-60.51 \pm 4.67$ & $-59.99 \pm 3.92$ & \multirow[t]{2}{*}{ 8. Somatic AP spike count } & $1 \pm 0$ \\
\hline & $-61.96 \pm 1.23$ & $-61.63 \pm 1.28$ & $-60.38 \pm 1.43$ & & $1 \pm 0$ \\
\hline \multirow[t]{2}{*}{ 9. Slow AHP time } & $0.238 \pm 0.030$ & $0.279 \pm 0.027$ & $0.213 \pm 0.037$ & 9. bAP amplitude at $620 \mu \mathrm{m}(\mathrm{mV})$ & $45 \pm 10$ \\
\hline & $0.225 \pm 0.039$ & $0.258 \pm 0.037$ & $0.215 \pm 0.054$ & $180 \mu \mathrm{m}$ below the BP & $33.39 \pm 7.91$ \\
\hline \multirow[t]{2}{*}{ 10. AP half-width (ms) } & $1.31 \pm 0.17$ & $1.38 \pm 0.28$ & $1.86 \pm 0.41$ & 10. bAP amplitude at $800 \mu \mathrm{m}(\mathrm{mV})$ & $36 \pm 9.33$ \\
\hline & $0.96 \pm 0.04$ & $0.96 \pm 0.04$ & $0.96 \pm 0.04$ & at the $\mathrm{BP}$ & $23.65 \pm 7.72$ \\
\hline
\end{tabular}

Table S2: Acceptable models match the empirically observed mean and standard deviation 575 across features of dendritic and perisomatic physiology. Empirical data (grey) was adopted from Hay et al. $2011^{13}$. Please note: matching the means and standard deviations across models with those observed empirically across L5PTs was not an objective during the parameter space exploration. Remarkably, the distributions of parameters across all acceptable models resemble nonetheless those observed empirically across L5PTs (i.e., similar means and standard deviations for most features). 